\title{
Ensinar a Gerência de Equipes em Disciplinas de
}

\section{Gerência de Projetos de Software}

\author{
Title: Teaching Team Management in Software Project Management Courses
}

\author{
Christiane Gresse von Wangenheim \\ GQS/INCoD/INE- Universidade \\ Federal de Santa Catarina (UFSC) \\ Campus Trindade - Florianópolis - \\ $\mathrm{SC}-$ Brasil \\ gresse@inf.ufsc.br
}

\author{
Osório Pereira Carvalho \\ GQS/INCoD/INE- Universidade \\ Federal de Santa Catarina (UFSC) \\ Campus Trindade - Florianópolis - \\ $\mathrm{SC}$ - Brasil \\ osorio.carvalho@gmail.com
}

\author{
Paulo Eduardo Battistella \\ GQS/INCoD/INE- Universidade \\ Federal de Santa Catarina (UFSC) \\ Campus Trindade - Florianópolis - \\ $\mathrm{SC}$ - Brasil \\ paulo@incod.ufsc.br
}

\begin{abstract}
Resumo Atualmente existe uma grande necessidade de formação de profissionais de software com competência em gerência de projetos. Para isso, a aprendizagem de habilidades interpessoais é indispensável. Porém, basicamente não existem atividades educacionais voltadas ao ensino de habilidades interpessoais em cursos de computação. Dentro desse contexto, o presente trabalho apresenta uma dinâmica para ensinar as dificuldades encontradas na gerência de equipes, em projetos de software. A dinâmica foi desenvolvida de forma sistemática seguindo um processo de design instrucional, sendo aplicado e avaliado em duas disciplinas de graduação na computação da UFSC em 2012-1. Os primeiros resultados indicam que a dinâmica pode ter um impacto positivo na motivação, experiência do usuário e aprendizagem dos alunos.
\end{abstract}

Palavras-Chave: Jogos Educativos, Engenharia de Software, Ensino e Aprendizagem

\begin{abstract}
Currently there exists a great need to teach project management competence to software professionals. Therefore, learning interpersonal skills is essential. But, there exist, basically, no educational activities aimed at teaching interpersonal skills in computing courses. Within this context, this paper presents an educational activity to teach the difficulties encountered in managing software project teams. The activity has been developed systematically following the instructional design process and has been applied and evaluated in two undergraduate computing courses at UFSC in 2012-1. First results indicate that the activity can have a positive impact on the student's motivation, user experience and learning.
\end{abstract}

Keywords: Educational Games, Software Engineering, Teaching and Learning 



\section{Introdução}

No crescente cenário do mercado nacional de software [1], fica evidente a necessidade de qualificação dos profissionais da área, inclusive na formação de gerentes de projeto [2]. O gerente de projetos é o profissional designado pela organização para atingir os objetivos do projeto [10]. Ele é o responsável pela comunicação entre as partes interessadas, ocupando o centro das interações entre estas e o projeto, trabalhando de maneira especial com a equipe de projetos. Ele participa da escolha dos membros da equipe e os lidera em busca dos resultados esperados [4]. Entre as suas atribuições destacamos: identificar as necessidades do projeto, estabelecer objetivos, equilibrar as demandas conflitantes de qualidade, escopo, tempo e custo, entre outras [10]. Um gerenciamento de projeto eficaz exige do gerente de projetos várias competências, incluindo: conhecimento sobre gerência de projeto e de administração em geral, conhecimento sobre normas e regulamentos da área de aplicação, entendimento do ambiente de projeto e habilidades interpessoais [10].

Entre as competências necessárias ao gerenciamento de projetos, destacamos as habilidades interpessoais, conhecidas também como soft skills [10]. Isso tipicamente envolve habilidades de liderança, comunicação, motivação, gerência de conflitos e resolução de problemas. No gerenciamento de projetos algumas dessas habilidades interpessoais se destacam devido a sua importância para o sucesso do projeto, tendo sua relevância reconhecida pelas organizações brasileiras [13]. No entanto, essas mesmas organizações consideram essas habilidades ainda deficientes em seus profissionais de gerenciamento de projetos [2], [13].

Isso demonstra a necessidade de abordar essas habilidades na formação acadêmica de gerentes de projeto. Entretanto, podemos observar que o ensino de gerenciamento de projetos, a nível acadêmico ou em forma de treinamentos, está fundamentado no conhecimento teórico, com pouca ou nenhuma atividade prática [9]. Por sua vez, o ensino de habilidades interpessoais recebe um foco ainda menor [14]. Porém, existem treinamentos na área de gerência em geral focando, por exemplo, o treinamento de liderança, a resolução de conflitos, entre outros. $\mathrm{O}$ problema é que esses treinamentos são generalizados, não apresentando uma aplicação dos conceitos na gerência de projetos de software [8]. Surge então a necessidade de incorporar a experiência prática à estratégias de ensino como jogos, dinâmicas, dramatizações, etc., [9] para integrar o ensino de habilidades interpessoais nas disciplinas de computação.

Com o intuito de estudar a aplicabilidade de atividades educacionais para o ensino de habilidades interpesso- ais em disciplinas de computação, o presente artigo apresenta um estudo de caso que aplica uma dinâmica para ensinar a importância das habilidades interpessoais no processo de gerenciamento de equipe, em projetos de software. A dinâmica é voltada à disciplinas de Gerência de Projetos de Software, no âmbito de cursos presenciais de graduação na área de computação, em universidades públicas brasileiras.

\section{Metodologia}

O presente trabalho foi desenvolvido como pesquisa exploratória usando uma abordagem de pesquisa multimétodos dividida em 4 etapas:

Etapa 1 - Análise da literatura. Nesse primeiro passo a literatura foi analisada em relação à atividades educacionais existentes e ao ensino de habilidades interpessoais.

Etapa 2 - Desenvolvimento da atividade educacional. Seguindo o processo de design instrucional ADDIE [7] foi desenvolvida sistematicamente uma atividade instrucional. Em um primeiro passo foi realizada a definição da meta instrucional, a análise dos aprendizes e do contexto, a análise instrucional e a definição dos objetivos de desempenho. A partir disso foi projetada a atividade educacional, desenvolvendo deste modo a estratégia instrucional. Em seguida foram desenvolvidos os materiais instrucionais.

Etapa 3 - Aplicação e avaliação da atividade educacional. A atividade educacional foi avaliada por meio de um estudo de caso dentro de duas disciplinas de Gerência de Projetos em cursos de graduação de computação na Universidade Federal de Santa Catarina (UFSC) em 2012-1. O estudo de caso foi sistematicamente definido, planejado, executado e analisado seguindo o processo proposto por Wohlin [15], e avaliado a partir do modelo de avaliação de jogos educacionais na área de Engenharia de Software proposto por Savi [12].

\section{A Dinâmica: Lidar com Pessoas Di- fíceis}

O objetivo da dinâmica "Lidar com Pessoas Difíceis" é ilustrar as dificuldades relacionadas à gerência de equipes em projetos de software. Durante esta atividade, os alunos em grupo simulam uma reunião do kick-off de um projeto de software. Um dos alunos assume o papel de gerente de projeto, que conduz a reunião e ao final precisa ter recebido as assinaturas de todos os membros do grupo firmando comprometimento com o projeto. O problema é que cada um dos outros membros do grupo (representando os membros do projeto de software) recebe uma caracterização de uma personalidade difícil (p.ex. explodindo com facilidade, reclamando de tudo, sabendo 


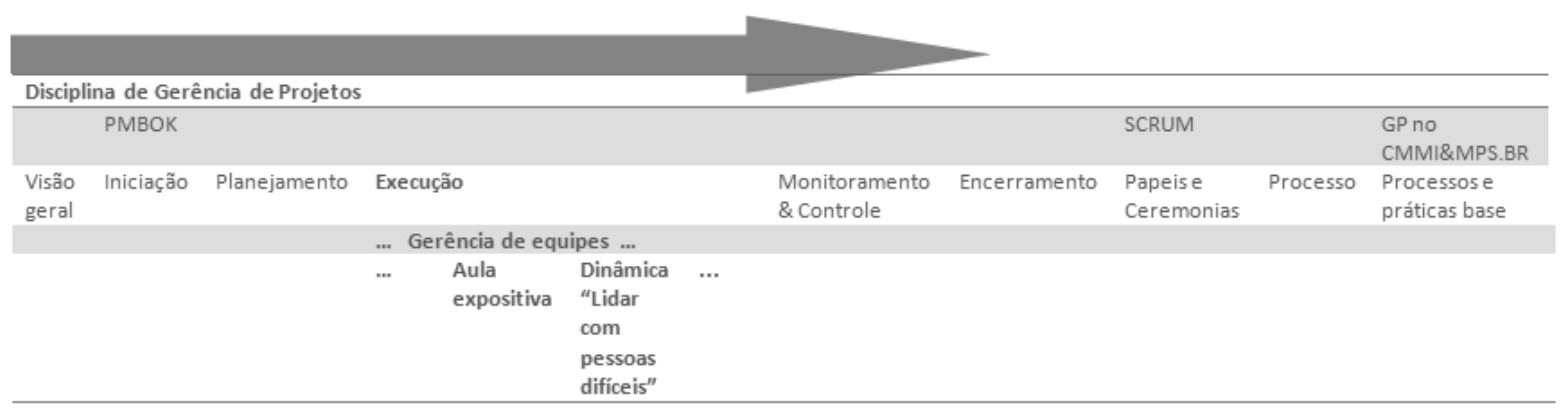

Figura 1: Inserção da dinâmica no fluxo da disciplina/unidade instrucional.

tudo melhor, etc.) baseado em Brinkmann e Kirschner [3] e deve agir de acordo com ela até o momento em que o gerente de projeto começa reagir de forma adequada. A partir desse momento o membro deve se tornar uma pessoa cooperativa que se compromete com o projeto.

A meta instrucional da atividade é: alunos do curso de graduação na computação (Ciência da Computação ou Sistemas de Informação) devem ser capazes de reconhecer a dificuldade (e consequentemente a importância de habilidades interpessoais) para gerenciar equipes em projetos de software. Especificamente em termos de objetivos de desempenho, esperamos que após a execução da dinâmica os alunos sejam capazes de:

- perceber os variados tipos de personalidades existentes, especificamente as chamadas pessoas difíceis, particularmente dentro de uma equipe de projetos, e

- reconhecer as dificuldades encontradas na prática da gerência de equipes em projetos de software.

Dessa forma, a atividade não tem a pretensão de ensinar aos alunos como gerenciar equipes, mas sim torná-los mais conscientes e atentos quanto a questão de gerência de equipes.

Publico alvo: A atividade se destina primeiramente aos alunos de graduação na área de computação. Um prérequisito para a aplicação da atividade é que os participantes tenham conhecimento básico sobre o processo de gerência de projetos.

Contexto: A dinâmica é projetada para ser aplicada durante aulas das disciplinas de Gerência de Projetos, dentro do currículo dos cursos de graduação na área de computação. Para possibilitar a ampla aplicação em universidades brasileiras, visamos o projeto de uma atividade com baixo custo, na língua portuguesa.

A atividade é prevista para ser aplicada dentro de uma unidade instrucional que aborda o grupo de processos de execução do projeto, incluindo o processo de gerência de equipes (Figura 1). A unidade instrucional na execução de projetos engloba todos os processos relacionados ao PMBOK [11], incluindo também o processo de gerência de equipes. Para ensinar esse processo, num primeiro momento o conhecimento teórico é apresentado por meio de aula expositiva. Em seguida é aplicada a dinâmica "Lidar com pessoas difíceis" para ilustrar as dificuldades encontradas na execução desse projeto na prática. A aplicação da dinâmica segue os passos apresentados na Tabela 1 .

\begin{tabular}{l|l}
\hline \multicolumn{1}{c|}{ Passo } & \multicolumn{1}{c}{ Tempo } \\
\hline Explicação da dinâmica & 10 minutos \\
\hline Execução da dinâmica & 15 minutos \\
\hline Discussão/Debriefing & 5 minutos \\
\hline
\end{tabular}

Tabela 1: Passos da dinâmica "Lidar com pessoas difíceis".

O material da dinâmica consiste em um conjunto de sete cartas de personalidades difíceis, uma tabela listando todas as personalidades e uma carta resumindo o projeto de software.

As cartas de personalidade apresentam as informações referentes a uma das personalidades difíceis seguindo Brinkmann e Kirschner [3], incluindo instruções de como a pessoa deve se comportar representando essa personalidade e informações sobre como o gerente deve reagir de forma correta (Figura 2).

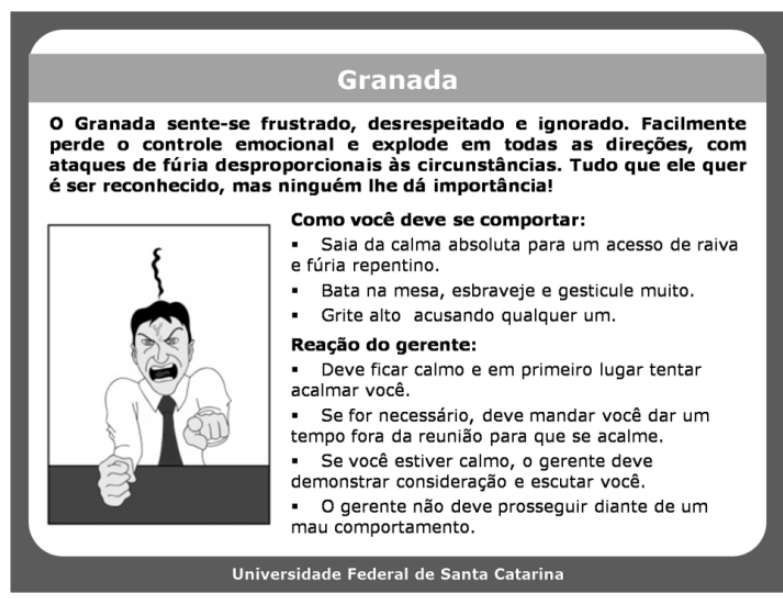

Figura 2: Exemplo de uma carta de personalidade.

A tabela de personalidades lista todas as personalida- 
des (Figura 3). O objetivo da tabela é auxiliar o aluno atuando como gerente no reconhecimento da personalidade que está sendo representada, além de guiá-lo na reação adequada personalidades difíceis para ao final conquistar o comprometimento da equipe.

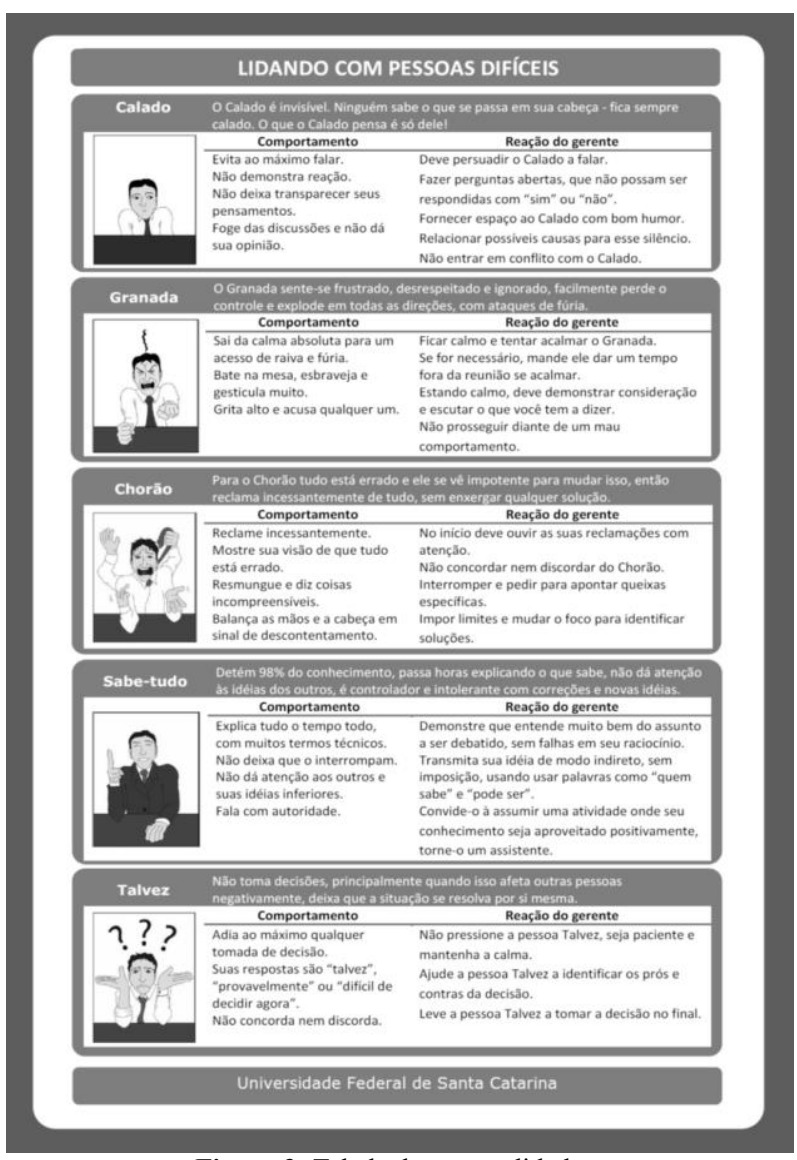

Figura 3: Tabela de personalidades.

A carta resumindo o projeto de software (Figura 4) descreve o projeto a ser discutido durante a reunião. As informações contidas nessa carta são apresentadas pelo gerente no início da reunião aos demais participantes. A carta apresenta também um espaço destinado à coleta de assinaturas dos participantes.

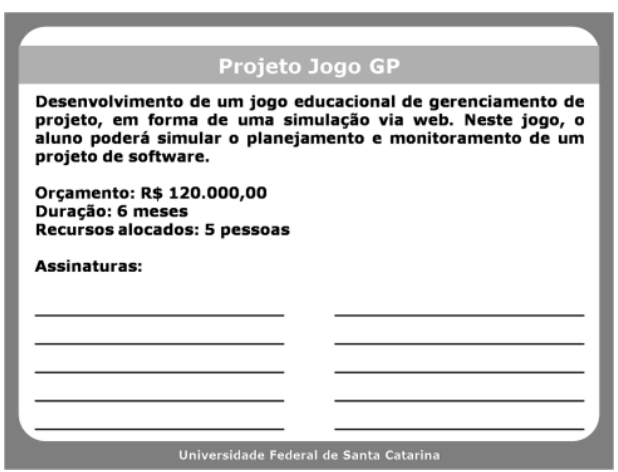

Figura 4: Carta do projeto.

Também fazem parte da dinâmica, dois conjuntos de slides com informações necessárias para explicação da dinâmica e para guiar a discussão/debriefing após a simulação da reunião. Todo o material da dinâmica está publicamente disponível em Português no site do GQS/INCoD/INE/UFSC (www.gqs.ufsc.br) sob a licença Creative Commons [5].

\section{Aplicação e Avaliação}

A aplicação e avaliação da dinâmica "Lidar com pessoas difíceis" foi realizada na aula sobre a execução de projetos na disciplina INE5427 - Planejamento e Gestão de Projetos do curso Ciência da Computação/INE/UFSC (com 14 alunos) e na disciplina INE5617 - Gerência de Projetos do curso de Sistemas de Informação/INE/UFSC (com 28 alunos) no semestre 2012-1 (Figura 5).

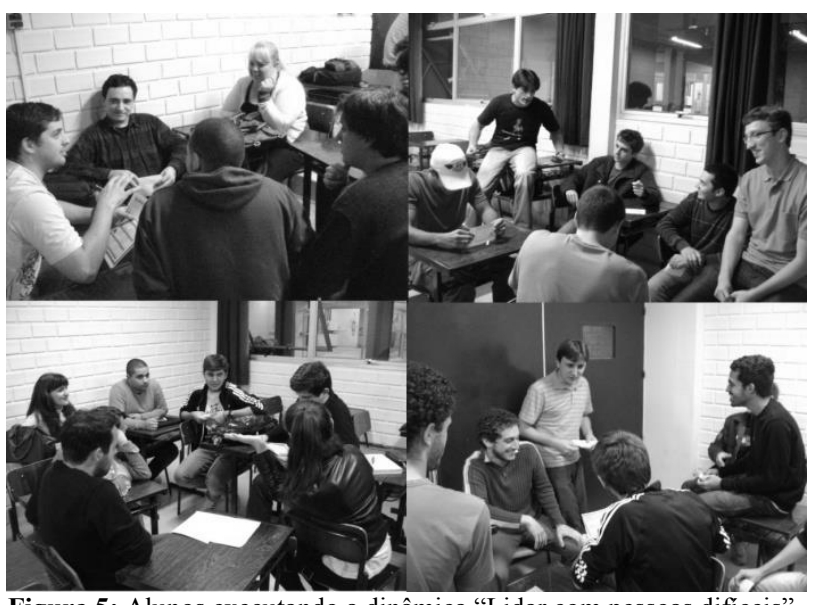

Figura 5: Alunos executando a dinâmica "Lidar com pessoas difíceis".

Nesta etapa da disciplina os alunos já obtiveram uma compreensão básica sobre os conceitos e o processo geral de gerência de projetos.

\subsection{Definição da avaliação}

O objetivo da avaliação é determinar se a dinâmica "Lidar com pessoas difíceis" possibilita alcançar os objetivos de aprendizagem e se ela, como estratégia instrucional, provoca a motivação dos alunos com uma boa experiência do usuário.

Para atingir esse objetivo avaliamos a dinâmica por meio de um estudo de caso no nível 1 de avaliação, seguindo o modelo de Kirkpatrick e Kirkpatrick [6] usando um design não-experimental (one-shot post-test only).

Como modelo de avaliação usamos o modelo de avaliação de jogos educacionais proposto por Savi [12], que engloba avaliação da motivação, experiência do usuário e impacto na aprendizagem. Os dados foram coletados usando o questionário padronizado [12] numa escala Likert de 5 pontos, que varia entre "Discordo Fortemente" (-2) até "Concordo Fortemente" (+2). 


\subsection{Análise dos dados}

Os dados coletados foram transferidos para planilhas do Microsoft Excel, que compõe o modelo de avaliação [12] e desse modo, foram gerados gráficos que apresentam a mediana da tendência central do conjunto de valores analisados.

\section{Avaliação do sub-componente Motivação}

De modo geral, as questões relacionadas à motivação obtiveram uma boa avaliação, como pode ser observado no gráfico apresentado na Figura 6. Apenas a questão relacionada ao esforço pessoal para avançar na dinâmica e a atratividade do design da dinâmica obtiveram, em uma das aplicações, uma mediana com valor zero. A grande maioria dos itens alcançou resultados que variam entre 1 e 2 . Os itens que obtiveram as melhores avaliações foram: a variação da dinâmica, a relevância do conteúdo para os interesses dos participantes. Assim como as categorias que obtiveram as melhores avaliações foram: a relevância e a confiança.

\section{Avaliação do sub-componente Experiência do Usuário}

A análise da experiência do usuário apresentou uma grande variação nos resultados, ainda assim, a maioria das pontuações oscilou entre 1 e 2 , conforme mostra o gráfico apresentado na Figura 7. A dimensão melhor avaliada foi a interação social. Entre os itens, tiveram destaque positivo a interação com outras pessoas durante a dinâmica e o esquecimento das preocupações durante a concentração na dinâmica, ambos com valor 2 nas duas aplicações. Por outro lado, o item "quando interrompido fiquei desapontado que a dinâmica tinha acabado" teve o ponto neutro como resultado. No entanto, os demais itens da dimensão nomeada como diversão foram bem avaliados, tendo o item "me diverti com a dinâmica" alcançado as maiores pontuações.

\section{Avaliação do sub-componente Aprendizagem}

As questões sobre o impacto de aprendizagem obtiveram mediana 1 em ambas as aplicações (Figura 8), indicando um impacto positivo na aprendizagem percebido pelos alunos.

A análise da dinâmica "Lidar com pessoas difíceis" demonstrou uma boa aceitação dos alunos ao propor a percepção das diferentes personalidades consideradas difíceis e das dificuldades causadas por essas personalidades no gerenciamento de projetos de software. Sua aplicação no apoio ao ensino do processo de gerenciar a equipe se mostrou positiva, de acordo com os resultados da avaliação. Em termos de motivação é possível perceber que a dinâmica prendeu a atenção dos alunos e que eles a consideraram relevante para seu aprendizado.

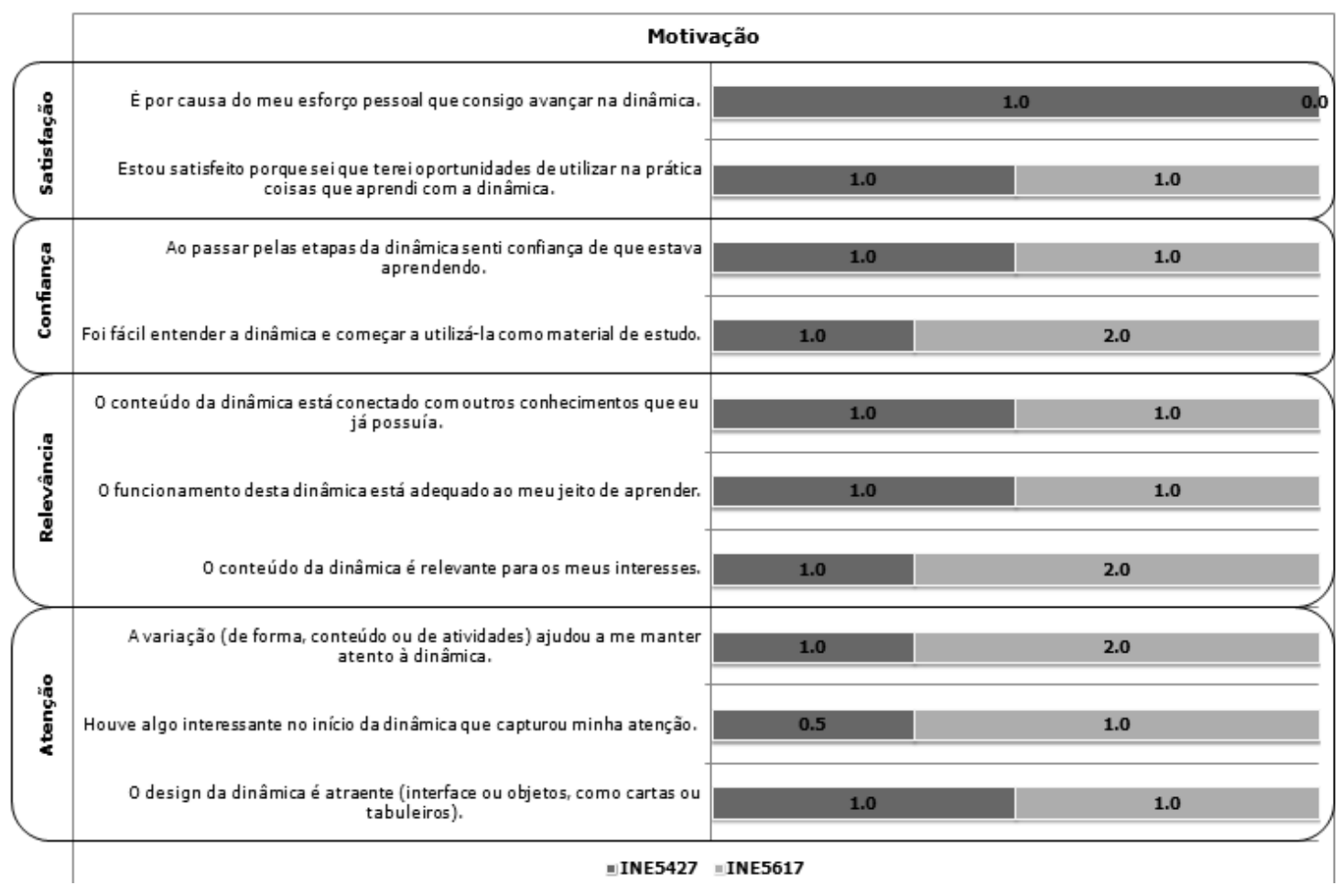

Figura 6: Mediana dos itens referentes à motivação. 


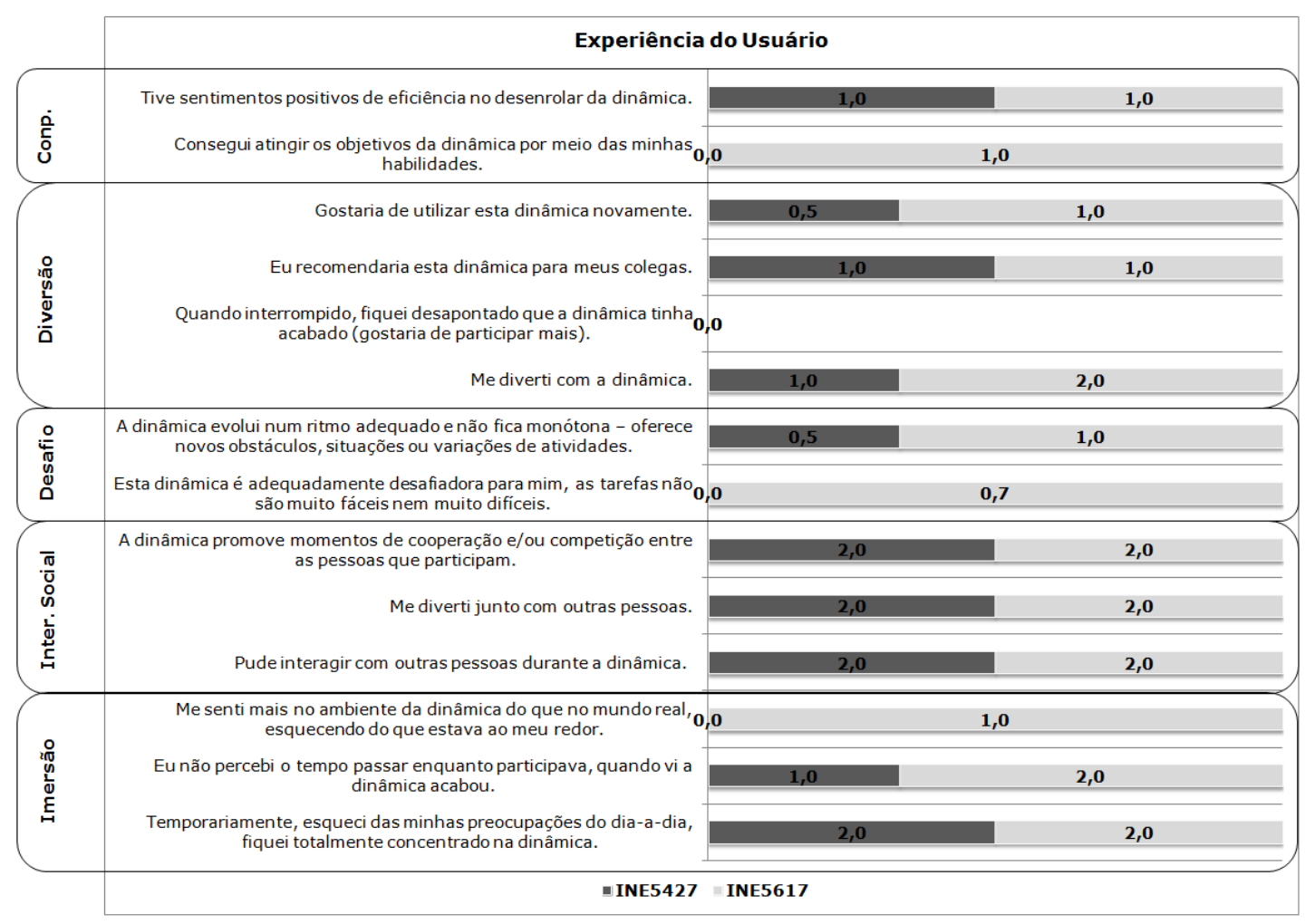

Figura 7: Mediana dos itens referentes à experiência do usuário.

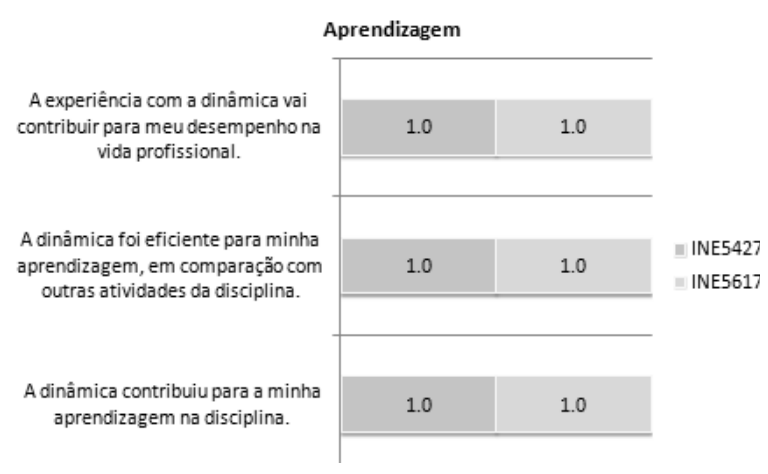

Figura 8: Mediana dos itens referentes à aprendizagem.

Referente a experiência do usuário a dinâmica se destacou em relação à interação social e à imersão, seguidos pelo desafio, compreensão e diversão. Esse é um resultado importante, principalmente no contexto de uma atividade relacionada a habilidades interpessoais.

Poucos itens receberam pontuações abaixo de zero. As questões que obtiveram notas mais baixas, em conjunto com os comentários deixados pelos participantes nos questionários aplicados, apontam melhorias a serem feitas para aperfeiçoar ainda mais a dinâmica.
Ameaças à validade. Como qualquer estudo empírico os resultados obtidos na presente avaliação precisam ser interpretados com cuidado levando em consideração ameaças à sua validade. Entre os fatores que a ameaçam, estão a falta de comparação com outras atividades similares com os mesmos propósitos, a partir de um estudo empírico com caráter não-experimental. Outro fator é a falta de uma medida efetiva do nível de conhecimento, antes e depois da aplicação da dinâmica, para medir o real aprendizado dos participantes. Outra questão que pode ter impacto na validade dos resultados - principalmente para generalização dos resultados - é o tamanho da amostra incluindo um número relativamente pequeno de alunos em apenas duas aplicações, ambos na mesma instituição. Outro fator importante é que a coleta de imagens durante as aplicações, com fotografias e filmagens, pode ter inibido os participantes e impactado na avaliação. Para evitar esse fator devem ser feitas novas avaliações sem gravações ou fotografias. Por último, a aplicação do questionário, que pode também ter intimidado os participantes, embora tenha sido devidamente explicado a finalidade da avaliação da dinâmica, assim como a participação anônima. 


\section{Conclusão}

Esse artigo apresenta o desenvolvimento, aplicação e avaliação de uma dinâmica para ensinar as dificuldades encontradas no processo de gerência de equipes, em projetos de software, assim como a importância de habilidades interpessoais na solução das mesmas. Os benefícios alcançados se resumem a própria dinâmica como ferramenta de apoio ao ensino de gerenciamento de projetos para alunos de cursos da área de computação de universidades brasileiras, simulando dentro da sala de aula parte da realidade a ser encontrada pelos estudantes no mercado de trabalho. Os resultados fornecem uma primeira indicação que a dinâmica pode ter um impacto positivo em termos de motivação, experiência do usuário e aprendizagem dos alunos. Outra vantagem da dinâmica é a disponibilidade do material em Português, tendo um custo muito baixo de confecção do material e assim permitindo uma ampla utilização em instituições públicas.

\section{Agradecimentos}

Os autores gostariam de agradecer Iury Givago Ribeiro Bispo de Almeida Santos por criar e disponibilizar os desenhos das pessoas difíceis para uso nesta dinâmica. Gostaríamos também agradecer todos os alunos que participaram das aplicações por fornecer o feedback.

Este trabalho foi apoiado pelo CNPq, uma entidade do governo brasileiro com foco em desenvolvimento científico e tecnológico.

\section{Referências}

[1] ABES. Mercado Brasileiro de Software: Panorama e Tendências. 2010. São Paulo. http://www.abes.org.br/templ3.aspx? $\mathrm{id}=306 \& \mathrm{su}$ $\underline{b}=596$

[2] ACATE. Relatório de Mapeamento dos Recursos Humanos e Cursos de TIC em Santa Catarina - Edição 2011. http://www.acate.com.br/index.asp?dep=7\&pg= 7879

[3] R. Brinkman e R. Kirschner. Aprendendo a lidar com pessoas difíceis. Rio de Janeiro: Gmt Editores Ltda, 2006. 80 p.

[4] G. Camarin e V. J. Sousa. As Habilidades do Gerente de Projetos: Um Fator de Sucesso para as Organizações. Revista Eletrônica de Administração, UFRGS, Porto Alegre/RS, 12(4), 2006.

[5] O. P. Carvalho. Objeto de aprendizagem para apoiar o ensino de habilidades interpessoais no gerenciamento de projetos de software. TCC - Sistemas de Informação/INE/UFSC, Florianópolis/SC, 2012.

[6] D. L. Kirkpatrick e J. D. Kirkpatrick. Evaluating Training Programs: The Four Levels. BerrettKoehler Publishers; 3rd ed., 2006.

[7] M. Molenda, C. M. Reigeluth e L. M. Nelson. Instructional Design. In: Encyclopedia Of Cognitive Science, London, 2003.

[8] C. G. von WANGENHEIM e F. SHULL. To Game or not to Game?. IEEE Software, 26(2), 2009.

[9] R. Prickladnicki e C. G. von Wangenheim. O Uso de Jogos Educacionais para o Ensino de Gerência de Projetos de Software. In: SBES 2008 - Fórum de Educação em Engenharia de Software (FEES), Campinas/SP, 2008.

[10] PMI (Org.). GUIA PMBOK - Um Guia do Conjunto de Conhecimentos em Gerenciamento de Projetos. 3. ed. Pennsylvania/EUA, 2004.

[11] PMI (Org.). GUIA PMBOK - Um Guia do Conhecimento em Gerenciamento de Projetos. 4. ed. Pennsylvania/EUA, 2008.

[12] R. SAVI. Avaliação de jogos voltados para a disseminação do conhecimento. 2011. $236 \mathrm{f}$. Tese (Doutorado) - Universidade Federal de Santa Catarina (UFSC), Florianópolis, 2011.

[13] PROJECT MANAGEMENT INSTITUTE CHAPTERS BRASILEIROS (PMI-CB) (Brasil). Estudo de Benchmarking em Gerenciamento de Projetos Brasil 2010: Relatório Geral. Rio de Janeiro, 2010. Disponível em: $<$ http://www.pmsurvey.org/>. Acesso em: 10 jun. 2012.

[14] SOCIEDADE BRASILEIRA DE COMPUTAÇÃO (Brasil). Currículo de Referência da SBC para Cursos de Graduação em Computação e Informática. Porto Alegre, 2003.

[15] C. WOHLIN et al. (2000). Experimentation in Software Engineering: An Introduction, Springer, Berlin. 